\title{
A methodology for energy key performance indicators analysis
}

Pedro Faria ${ }^{1,2}$, Fernando Lezama ${ }^{1,2}$, Zita Vale $^{2^{*}}$ (D) and Mahsa Khorram ${ }^{1,2}$

\author{
* Correspondence: email.zitavale@ \\ gmail.com \\ ${ }^{2}$ Polytechnic of Porto, Porto, \\ Portugal \\ Full list of author information is \\ available at the end of the article
}

\begin{abstract}
With the advent of the smart grid era, the electrical grid is becoming a complex network in which different technologies coexist to bring benefits to both customers and operators. This paper presents a methodology for analyzing Key Performance Indicators (KPIs), providing knowledge about the performance and efficiency of energy systems, focusing on the demand side. In the first stage of the methodology, the baseline KPIs are calculated. In the second stage, all KPIs are updated to be compared with the baseline ones. In fact, due to the dynamic nature of players in a smart grid, this methodology plays a crucial role in the performance assessment. Moreover, the proper definition and selection of KPIs is usually a challenging task since KPIs can be applied to evaluate diverse areas within a smart grid. Such areas include building energy efficiency, home communications, and smart metering deployment, just to mention a few. In the proposed methodology, the information obtained from a KPI can be driven to distinct aspects such as efficiency, environment, reliability, power quality, safety, security, or cost reduction. Through a case study from a real implementation of an energy system, we show how to assess energy consumption efficiency, thus improving energy management.
\end{abstract}

Keywords: Analysis tools, Metrics, KPIs, Smart grids

\section{Introduction}

Nowadays, electric grids are evolving fast into complex systems mainly driven by smart grid (SG) technologies. Different technologies coexist in this dynamic context to bring benefits to both customers and operators (Lezama et al. 2019). Due to the multidisciplinary nature of technologies to be considered in an SG, specialized tools with the capacity of evaluating key performance indicators (KPI) are needed to validate and demonstrate the value of modern solutions applied to problematics in power systems (Abrishambaf et al. 2015).

However, the proper definition and selection of KPIs is usually challenging due to the variety of factors that may be considered. For instance, KPIs can be applied to evaluate specific areas within an SG, namely residential/building energy efficiency (Peng et al. 2013; Nistor and Antunes 2016), home communications (Li et al. 2015), smart metering deployment (Fan et al. 2013), just to mention a few. The information obtained from a KPI could be focused on different aspects,

(c) The Author(s). 2021 Open Access This article is licensed under a Creative Commons Attribution 4.0 International License, which permits use, sharing, adaptation, distribution and reproduction in any medium or format, as long as you give appropriate credit to the original author(s) and the source, provide a link to the Creative Commons licence, and indicate if changes were made. The images or other third party material in this article are included in the article's Creative Commons licence, unless indicated otherwise in a credit line to the material. If material is not included in the article's Creative Commons licence and your intended use is not permitted by statutory regulation or exceeds the permitted use, you will need to obtain permission directly from the copyright holder. To view a copy of this licence, visit http://creativecommons.org/licenses/by/4.0/. 
namely efficiency, environment, reliability, power quality, safety, security, or cost reduction (Personal et al. 2014).

As if that was not enough, KPIs must be measurable and verifiable and somehow used to reflect complex information as simple as possible for the involved parts (i.e., organizations and customers). Since SG technologies are designed to improve the current power delivery systems and increase the interaction between different stakeholders, initiatives and attempts have been proposed to assess the performance of solutions applied to power systems (Personal et al. 2014; May et al. 2015; González-Gil et al. 2015; Cabeza et al. 2015).

For instance, in the USA and some other countries, the International Performance Measurement and Verification Protocol (IPMVP) (IPMVP 2001) is an initiative (developed by a coalition of international organizations and the United States Department of Energy) that defines standard terms and suggests best common practices to determine energy and water savings associated with energy conservation measures. On the other hand, Personal et al. (Personal et al. 2014) proposed a new approach based on business intelligence to develop new metrics and KPIs for assessing its energy projects. The authors identified the multidisciplinary character of smart grids (involving various technologies) and recognized the challenging task of assessing the overall project goals. Another interesting approach is proposed by Gonzalez-Gil et al. for an urban rail system in (González-Gil et al. 2015). In that work, a holistic approach considering the numerous interdependencies between subsystems is used to take advantage of a comprehensive set of energy consumption related KPIs that enable a multilevel analysis of the actual energy performance of the system; an assessment of potential energysaving strategies; and the monitoring of the results of implemented measures. Similarly, Hanak et al. (Hanak et al. 2015) defined KPIs to estimate reliability indices based on the uncertainty of the input to a process model of a coal-fired power plant. A first attempt to collect organized KPIs used in thermal energy storage (TES) can be found in (Cabeza et al. 2015). The study is well-conducted; however, the authors only consider KPIs for TES in solar power plants (CSP) and buildings.

In this paper, a KPI assessment methodology focusing on SGs is provided. This is motivated by the need for a simple and easy-to-understand method to use KPIs in several applications, as most of the methods in the literature are based on calculations that the small electricity consumers and producers cannot easily understand.. First, we present a study on KPIs in power systems. The most suitable energy-related KPIs are identified from the literature review to make possible the abstraction of raw data from measurements of consumption and supplied power into quantitative indicators that better reflect the objectives pursued by stakeholders. Therefore, the scope is driven to power systems in general, namely smart grids, focusing on the consumption side. The developed methodology is used to determine a baseline and assess the impact of KPIs. Finally, a case study is used to illustrate the impact of photovoltaic (PV) generation in an energy community, representing a set of consumers with PV.

\section{Energy related KPI}

KPI can provide a broad range of useful information to validate the costs of investment, operation, and efficiency of a given solution or proposal. In the SG context, it is common to use KPIs to reflect in a quantitate manner specific aspects or objectives in a 
project, such as economic viability, environmental impact, reliability, and power quality. In the next subsections, some common indicators related to these goals are summarized.

\section{Economic viability}

The economic viability objective can be determined with the help of different indicators depending on the microgrid area to be assessed. For instance, in (Honarmand 2015), 5 second-class indicators, namely capital cost, replacement cost, maintenance cost, operation/generation cost, and power loss cost, are used to evaluate this objective.

a) Capital cost: this indicator should reflect the economic feasibility of a system, allowing the selection of microgrid implementations with the lowest capital cost (Yang et al. 2009):

$$
C_{\text {acap }}=C_{\text {cap }} \cdot \operatorname{CRF}\left(i R, Y_{\text {proj }}\right)=C_{\text {cap }} \cdot \frac{i R \cdot(1+i R)^{Y_{\text {proj }}}}{(1+i R)^{Y_{\text {proj }}}-1}
$$

where $C_{c a p}$ is the initial capital cost of a component, m.u.; $Y_{\text {proj }}$ is the component lifetime, month/year; $C R F$ is the capital recovery factor (i.e., a ratio to represent the value of an annuity); $i R$ is the interest rate which is related to the nominal interest rate and the annual inflation rate.

b) Replacement cost: This indicator represents the value of all the replacements costs occurring throughout the lifetime of a given project (Yang et al. 2009):

$$
C_{\text {arep }}=C_{\text {rep }} \cdot \operatorname{SFF}\left(i R, \mathrm{Y}_{\text {rep }}\right)=C_{\text {rep }} \cdot \frac{i R}{(1+i R)^{Y_{\text {rep }}}-1}
$$

where $C_{r e p}$ is the replacement cost of a component (e.g., a battery or metering), m.u.; $Y_{\text {rep }}$ is the component lifetime, month/year; SFF is the sinking fund factor (i.e., a ratio to represent the future value of a series of equal period cash flows); $i R$ is the interest rate.

iii) Maintenance cost: The system maintenance cost can be fixed as a constant, in m.u., every specified time (i.e., monthly or yearly depending on the duration of the project).

iv) Operation/generation cost: DGs generation costs are related to fuel consumption and fuel price of DGs that consume coal or gas (e.g., micro gas turbines and diesel engines):

$$
C_{\text {ope }}=\sum_{t=1}^{T} \sum_{i=1}^{N}\left[K_{f(i, t)} \cdot P_{D G(i, t)}+K_{o(i, t)} \cdot P_{D G(i, t)}\right]
$$

where $K_{f(i, t)}$ is the fuel coefficient of ith DG, m.u./kWh; $K_{o(i, t)}$ is the operation coefficient of ith DG, m.u./kWh; and $P_{D G(i, t)}$ is the power generated (in kW) by ith DG at time $t$. 
e) Power loss cost: Power loss indicators include both active and reactive power loss. Active power loss considers AC and DC components, namely AC transformers, $\mathrm{AC}$ distribution lines, DC converters and so on. Reactive power loss only refers to AC components (Honarmand 2015):

$$
\begin{aligned}
& \Delta P_{\text {active }}=\Delta P_{A C-\text { loss }}+\Delta P_{D C-\text { loss }}=\sum_{l=1}^{L} I_{l}^{2} R \\
& \Delta P_{\text {reactive }}=\sum_{l=1}^{L} I^{2} X
\end{aligned}
$$

\section{Environmental impact}

The objective related to the environmental impact requires indicators that measure greenhouse emissions produced by DGs units.

a) CO2 emission: in (Honarmand 2015) the following indicator to assess CO2 emission is suggested:

$$
E_{\text {ann }}=\sum_{t=1}^{T} \sum_{i=1}^{N} K_{i} \cdot M_{i} \cdot P_{D G(i, t)}
$$

where $K_{i}$ is the Emission coefficient of ith unit of DG, $\mathrm{kg} / \mathrm{kWh}$; and $M_{i}$ represents the Greenhouse gas emission cost, m.u./kg.

b) $\mathrm{CO} 2$ emissions in buildings with external suppliers consideration: when external suppliers are also considered, CO2 emissions (e.g., in buildings) can be modeled as (Soares 2017):

$$
E_{\text {ann }}=\sum_{t=1}^{T}\left(\sum_{i=1}^{N} P_{D G(i, t)} \cdot K_{i}+\sum_{s=1}^{S} P_{s p(s, t)} \cdot K_{s}\right)
$$

where $P_{s p(s, t)}$ is the power acquired throughout an external supplier s, $\mathrm{kW} ; K_{i}$ and $K_{s}$ are the CO2 emissions coefficients of DG and external suppliers, kgCO2/kWh.

\section{Reliability-related objectives}

Reliability indicators can be categorized into load point reliability and system reliability (Honarmand 2015). Load point indexes cover the following three aspects: i) Frequency of load interruptions (occurrence per year); ii) Average duration of load interruptions (period per occurrence): iii) Average annual duration of load interruptions (hours per year). On the other hand, a list of commonly system indexes includes (Grigsby 2012; Atwa and El-Saadany 2009): 
a) System Average Interruption Frequency Index (SAIFI):

$$
\mathrm{SAIFI}=\frac{\sum \lambda_{i} N_{i}}{\sum N_{i}}
$$

b) System Average Interruption Duration Index (SAIDI):

$$
\text { SAIDI }=\frac{\sum U_{i} N_{i}}{\sum N_{i}}
$$

iii) Average Service Availability Index (ASAI):

$$
\mathrm{ASAI}=\frac{T_{\text {total }} \cdot \sum N_{i}-\sum U_{i} N_{i}}{T_{\text {total }} \cdot \sum N_{i}}
$$

where $N_{i}$ is the number of consumers at load point I; $\lambda_{i}$ represents the failure rate at load point I; $U_{i}$ is the average duration of load interruption at load point I; $T_{\text {total }}$ is the number of hours considered for the assessment.

\section{Power quality}

Power quality objectives can be measured by three indicators, namely voltage qualified rate (VQ), power supplied from DGs $\left(P_{\text {sup }}\right)$ and total harmonic distortion (THD) (Hossam et al. 2014).

a) Voltage Qualified Rate: this indicator is related to the voltage limitations in electricity grids. Voltage deviations can be evaluated at each bus to judge the voltage quality $V_{q} \%$. For each node, the higher value of $V_{q} \%$ the better:

$$
V_{q} \%=\frac{\sum_{t=1}^{T} T_{q(t)}}{T}
$$

where $\Sigma T_{q} \%$ is the accumulated time that voltage is qualified, calculated as:

$$
T_{q(t)}=\left\{\begin{array}{c}
1, \text { if Vlower }<V_{(i, t)}<\text { Vupper } \\
0 \text { otherwise }
\end{array}\right.
$$

b) Power Supplied from DERs: this indicator reflects the total power generation, i.e. DGs and Energy storage systems: 


$$
P_{\sup (t)}=\sum_{t=1}^{T} \sum_{u=1}^{N_{D E R}} P_{D E R(u, t)}
$$

a) Total Harmonic Distortion: Harmonic distortions depend on both load levels and system conditions. Harmonic distortion can be defined as (Honarmand 2015):

$$
\begin{gathered}
V_{T H D}=\frac{\sqrt{\sum_{n-2}^{\infty} V_{n}^{2}}}{V_{1}} \\
I_{T H D}=\frac{\sqrt{\sum_{n-2}^{\infty} I_{n}^{2}}}{I_{1}}
\end{gathered}
$$

where $V_{n}$ and $I_{n}$ are the different time voltage and current, respectively.

\section{Related works}

The KPI subject has been analyzed in the literature for different scopes, motivating the work presented in this paper. In ( $\mathrm{Li}$ et al. 2017), different stakeholders are identified for the implementation of multi-level KPIs. The selection of KPIs is made for energy performance. In (Del Pero et al. 2018), the focus is given to energy storage in buildings, testing KPIs in several case-studies. Adding PV to the storage, the work in (Kourkoumpas et al. 2018) performs the life cycle analysis regarding stakeholders' needs. The building's perspective is addressed in (Al Dakheel et al. 2020), where KPIs are proposed to evaluate the performance of the building as a smart building.

Looking at the grid perspective, namely in the context of autonomous island grids, the work in (Pramangioulis et al. 2019) focuses on legal KPIs related to barriers for the implementation of smart grids. The overall smart grid economic and environmental evaluation is made in (Moretti et al. 2017), making a seventeen studies review where energy efficiency is included.

In a more general perspective, the work in (Van Gorp 2005) describes a method for the definition of KPIs driven to the success of energy management plans. Finally, in (Giordano et al. 2014), a European expert group defines a set of KPIs for the costbenefit analysis of smart grid projects.

The works mentioned above are mostly focused on KPIs specific for resources (PV and storage, for example), for buildings, for island grids, and for project evaluation. These works lack a simple approach that can be used to any kind of data, with clear identification of the baseline calculation phase. Also, the continuous evaluation of KPIs is an important aspect that deserved exploration in the literature. Finally, in the present paper, these aspects are combined with a case study of an energy community with different types of consumers and PV generation. 


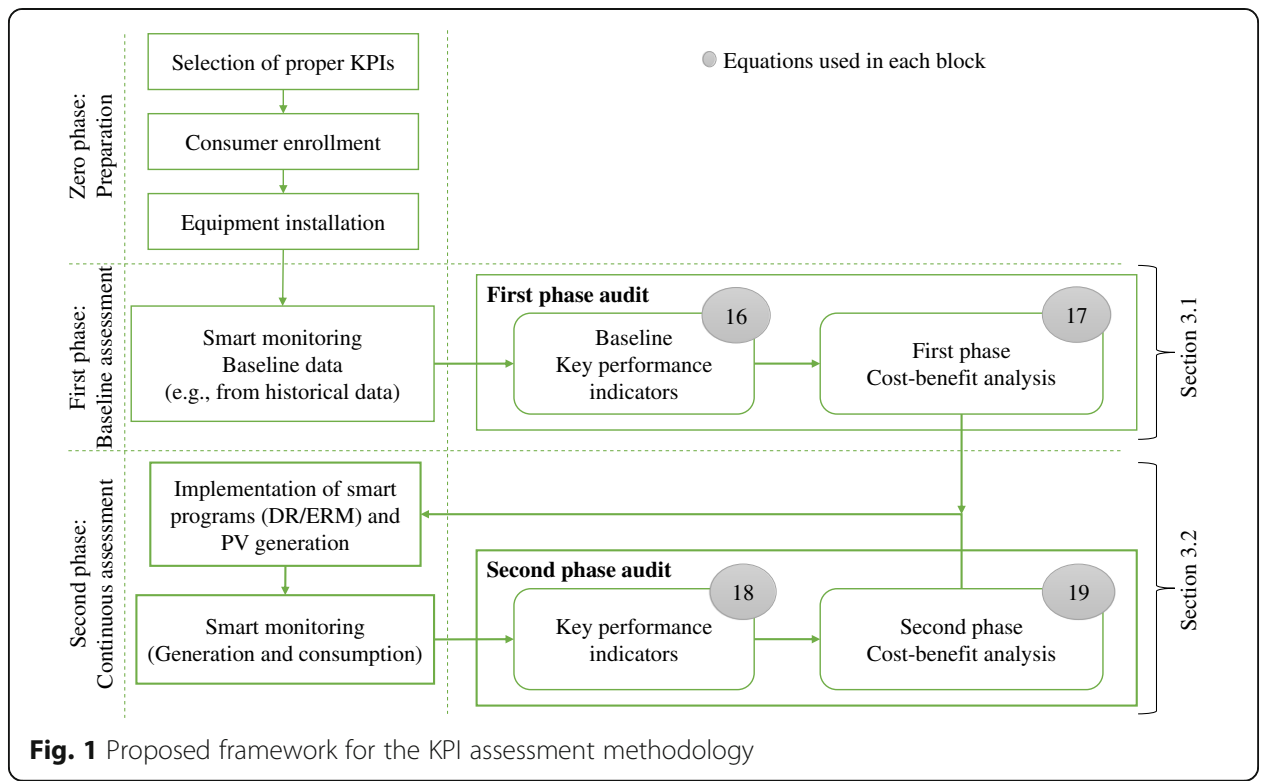

\section{KPIs and metodology used in this study}

Figure 1 presents a framework to evaluate the impact of energy management solutions and the use of KPIs. The methodology is composed of a preparation phase (i.e., zero phase) and a two-phase assessment. In the preparation phase (i.e., the zero phase), initial steps required to evaluate the proposed methodology are carried out. The zero phase includes the selection of the KPIs to be evaluated, ${ }^{1}$ the consumers' enrolment into the programs, and the equipment installation. Later, in the first phase of assessment, a baseline is constructed from the monitoring of selected buildings, and a costbenefit analysis is carried out. Finally, in the second phase, smart programs will be applied, and new data will be gathered to establish the impact of the analyzed programs. The second phase can be applied periodically to improve the performance of the system and bring benefits for both consumers and operators.

\section{Baseline KPIs}

The baseline is constructed using the information from the smart monitoring. This paper uses measurement data to calculate the annual average consumption patterns and monetary costs resulting from the application of different tariff types to different residential houses and buildings of an energy community.

The aggregated baseline load from different buildings is calculated as:

$$
L_{\text {agg }}=\sum_{n_{h}=1}^{H} \sum_{d=1}^{D} \sum_{t=1}^{T} L_{(h, d, t)}
$$

where $L_{(h, d, t)}$ is the load of house $h$, in day $d$, and time $t$. Therefore, $L_{\text {agg }}$ corresponds to the total load of a determined number of houses $(H)$, over a period of $D$ days, each day having $T$ measurements.

\footnotetext{
${ }^{1}$ The methodology can be applied to any set of KPIs. However, in this study, we limited the analyzed KPIs for easy comprehension of the results.
} 
Following the same logic, and having available the prices for different tariffs (Tariff $(t$, type $)$ the resulting baseline annual cost for different types of tariff is given by:

$$
\operatorname{Cost}_{\text {gen }(\text { type })}=\sum_{n_{h}=1}^{H} \sum_{d=1}^{D} \sum_{t=1}^{T} L_{(h, d, t)} \cdot \text { Tariff }(t, t y p e)
$$

The definition of tariff and the calculation of annual costs and aggregated loads is done using the available monitored measurements.

\section{IMPAC of installed PV generation}

After determining the baseline, current information resulting from a determined program can be used to perform a cost-benefit analysis. In particular, in the case study selected for this paper, it is desired to appreciate the impact of sharing PV generation in an energy community. To this end, PV generation capacity can be calculated as:

$$
P V_{g e n\left(n_{b}\right)}=\sum_{d=1}^{D} \sum_{t=1}^{T} P_{p v\left(d, t, n_{b}\right)}
$$

where $P_{p \nu(d, t, n b)}$ is the power generated by a PV unit installed in building $n b$, in day $d$, and time $t$.

Assuming that PV units are installed in specific buildings, the annual cost can be recalculated considering the self-consumption of the PV power as follow:

$$
\operatorname{Cost}_{w i t h P V\left(n_{b}, t y p e\right)}=\sum_{d=1}^{D} \sum_{t=1}^{T}\left[\left(L_{\left(d, t, n_{b}\right)}-P_{p v\left(d, t, n_{b}\right)}\right) \cdot \operatorname{Tariff}_{(t, t y p e)}\right]
$$

where $\operatorname{Cost}_{w i t h P\left(n_{b}, t y p e\right)}$ is the annual cost for building $n_{b}$ after using the selfconsumption of the PV generation for several types of tariffs.

With this second calculation, it is possible to provide a measurement of savings by comparing the baseline value and the impact of PV self-consumption as:

$$
\% \text { Savings }=\left[\left(\text { Cost }_{\text {baseline }}-\text { Cost }_{\text {pv }}\right) / \text { Cost }_{\text {base }}\right] * 100
$$

\section{Results and discussion}

In the current study, we use smart metering technology and real measurements from a case study of a community. We analyze the impact of different tariffs as well as the use of PV generation in the community. The result section is divided into four subsections as follows: 1) the case study is presented; 2) A baseline is set considering no PV generation; 3) the impact of using PV generation is reflected; 4) a comparison between the baseline and the impact of PV generation is highlighted. In this way, after presenting the case study, the application of the proposed methodology is presented in sub-section 3.1 in order to determine the baseline results. Then, using the procedure presented in sub-section 3.2, the PV generation impact in Municipal buildings is made as a selected example, where the KPIs are calculated along the time. Finally, as the last step of the proposed methodology, the conclusions of the audit are discussed, comparing the baseline with the updated KPI values. 


\section{Case study}

The selected case study corresponds to a network community. The network consists of 29 residential houses, ${ }^{2}$ and four public buildings, namely a public library, a municipal market, a culture house, and a city hall (Faria et al. 2019). The type of consumers and buildings in this case study has been chosen to match the case study in (Faria et al. 2019). The main purpose of this case study is to analyze data of interest to design and apply specific tariffs to these public and residential buildings and to identify the best use of available PV generation. To this end, the public buildings in the network community are equipped with a PV system.

It is considered that the library building has an electricity contract with a rate of 34.5 kVA, the Municipal market a contract of $41.4 \mathrm{kVA}$, and the City Hall electricity contract of $108 \mathrm{kVA}$. Moreover, these three buildings are equipped with PV panels with a maximum generation capacity of $17.28 \mathrm{~kW}, 9 \mathrm{~kW}$, and $8 \mathrm{~kW}$, respectively.

\section{Baseline results}

The house and buildings' energy consumption was monitored using available smart metering technology in intervals of $15 \mathrm{~min}$ for a period of 1 year (365 days). Figure 2 shows the daily average profile patterns for the 29 aggregated residential houses (Fig. 2a) and the public buildings (Fig. 2b). The aggregated load consumption from the residential houses reaches, on average, a power of $20 \mathrm{~kW}$, while the aggregated load from the public building presents a peak of around $30 \mathrm{~kW}$.

Referring to the Portuguese legislation (ERSE n.d.), five different tariffs are applied: 1) Simple; 2) Bi-hourly (day cycle); 3) Bi-hourly (week cycle); 4) Tri-hourly (day cycle); 5) Tri-hourly (week cycle). These tariffs are distinct in the way that they consider, respectively, the following: a single price for all the periods of the day; two different prices along different periods of the day, depending or not on the day of the week; and three different prices along different periods of the day, depending or not on the day of the week. Also, these tariffs consider only the energy component of the electricity bill. This means that it is ignored the network or distribution fees that each consumer must pay to the distribution system operator. The tariffs are applied to the data in order to calculate the annual cost for each building and the aggregated consumption of the residential houses. Table 1 presents such costs (calculated with Eq. 17), which are used as a baseline for further analysis.

\section{PV generation impact on municipal buildings}

In this section, the impact of the installation of PV generation is assessed. For this purpose, we analyze the generation of the PV systems installed in three public buildings, namely the library, the municipal market, and the cultural house.

Figure 3 shows the average daily PV generation of the installed PV systems in the public buildings and its impact on the average daily profile of the public buildings. It can be seen in Fig. 3a that the average peak generation power is around $13 \mathrm{~kW}$. With this generation capacity, Fig. $3 \mathrm{~b}$ shows that the average peak consumption of the public buildings can be reduced up to $22 \mathrm{~kW}$.

${ }^{2}$ Originally, 50 buildings from the community were considered for the case study. However, due to corrupted and missing data, only 29 buildings were considered in the final analysis. 


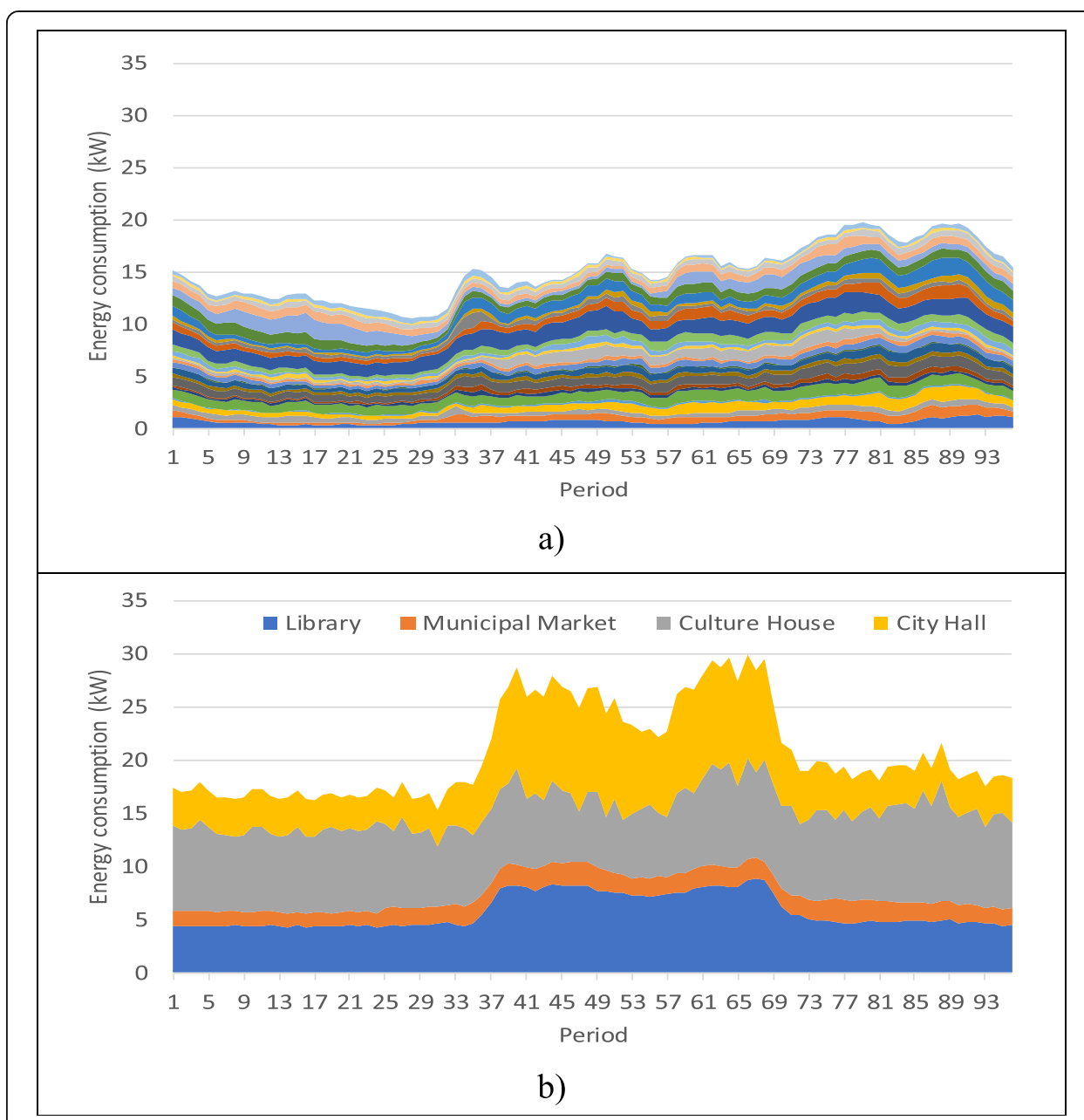

Fig. 2 Average energy consumption patterns. a Aggregated consumption of residential houses. $\mathbf{b}$ Aggregated consumption of public buildings

With this information, we can calculate the annual costs considering that the PV generation is self-used by the public building where the PV system is installed (Eq. 19). Table 2 presents the annual costs achieved for each public building when using their own PV generation. Moreover, we can calculate the percentage of savings (Eq. 20), showing that $27 \%$ of savings can be achieved using the tri-hourly (day cycle) tariff. In particular, the municipal market can obtain around $50 \%$ of costs saving using its PV system.

Table 1 Electricity annual cost with distinct types of tariffs (m.u.)

\begin{tabular}{llllll}
\hline & Simple & $\begin{array}{l}\text { Bi-hourly } \\
\text { (day cycle) }\end{array}$ & $\begin{array}{l}\text { Bi-Hourly } \\
\text { (week cycle) }\end{array}$ & $\begin{array}{l}\text { Tri-Hourly } \\
\text { (day cycle) }\end{array}$ & $\begin{array}{l}\text { Tri-Hourly } \\
\text { (week cycle) }\end{array}$ \\
\hline Agg. Houses & $85,740.19$ & $85,211.67$ & $82,461.05$ & $88,909.33$ & $81,494.68$ \\
Library & $32,497.62$ & $33,148.02$ & $31,578.31$ & $34,010.32$ & $32,110.05$ \\
Municipal Market & 9738.56 & 9737.47 & 9399.92 & $10,195.85$ & 9453.67 \\
Culture House & $43,796.15$ & $42,176.82$ & $40,975.70$ & $43,396.17$ & $40,255.56$ \\
City Hall & $31,666.96$ & $33,520.32$ & $31,721.48$ & $34,468.24$ & $32,341.51$ \\
Total Cost & $\mathbf{2 0 3 , 4 3 9 . 4 8}$ & $\mathbf{2 0 3 , 7 9 4 . 3 0}$ & $\mathbf{1 9 6 , 1 3 6 . 4 7}$ & $\mathbf{2 1 0 , 9 7 9 . 9 0}$ & $\mathbf{1 9 5 , 6 5 5 . 4 8}$ \\
\hline
\end{tabular}




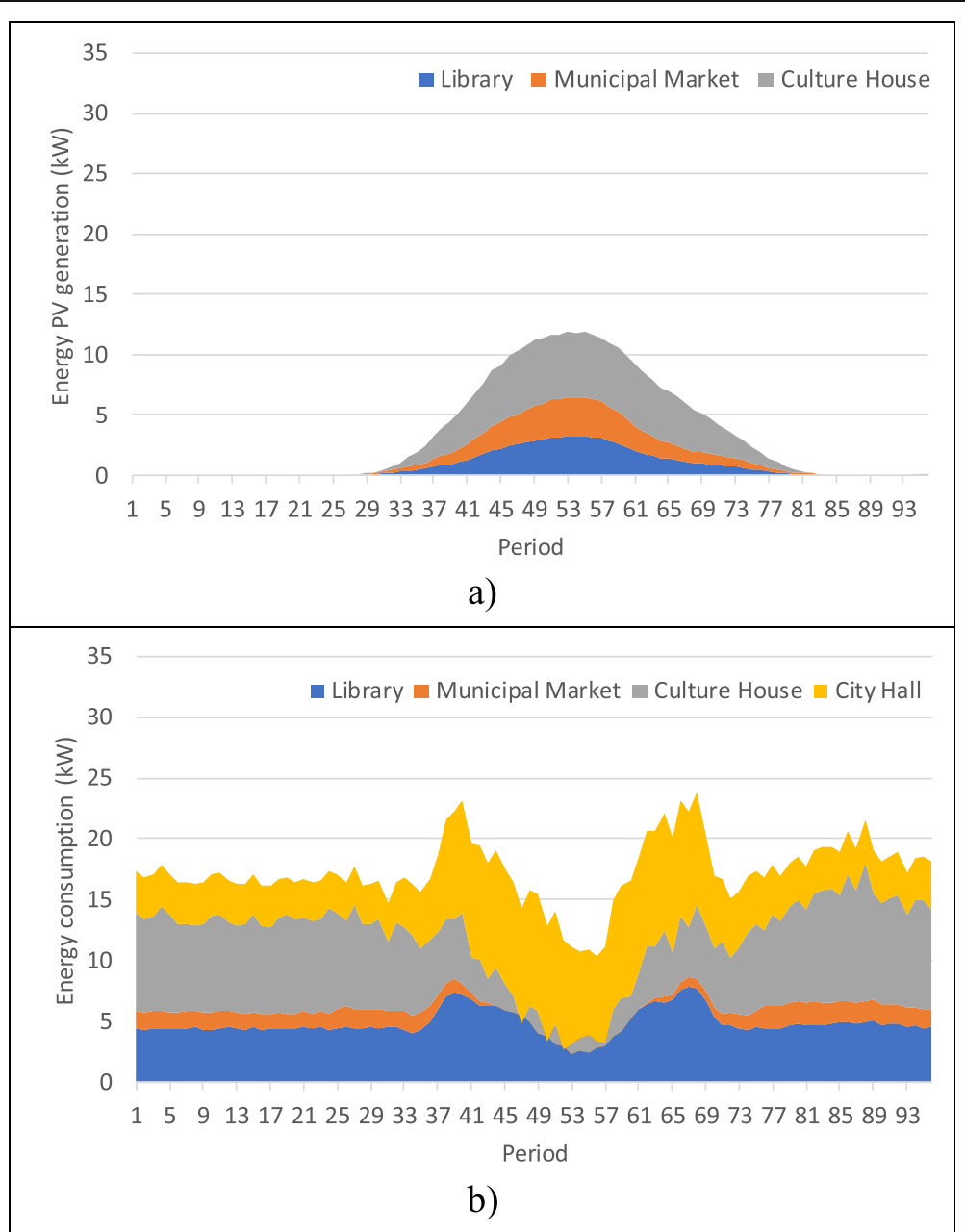

Fig. 3 Average PV generation and the impact in self-consumption of public buildings. a Aggregated PV generation. b Aggregated consumption of public buildings after using PV generation

Figure 4 shows the PV generation from the public buildings and their impact on the aggregated average profile of the residential buildings. As shown in Fig. 4b, the use of the PV generation on the houses does not contribute to a reduction of the peak power. This is mainly due to the peak power on this case study for the residential houses is presented on the periods belonging at night times. Therefore, the influence of the PV generation is neglected on the reduction of peak power consumption.

However, we can also analyze the impact of PV generation on the annual cost of residential houses. Table 3 presents the total costs and percentage of savings for the residential houses if the PV generation from public buildings is used for this end. As can be seen, by using the PV generation and the Bi-hourly tariff, a total annual percentage saving of around $27 \%$ can be achieved.

\section{Comparison between baseline and PV generation usage}

Making use of the proposed methodology, namely using equations eq. (18), eq. (19) and eq. (20), presented in sub-section 3.2, it has been made the study of the PV 
Table 2 Electricity Annual cost and percentage of savings for public buildings with distinct types of tariffs considering PV generation (m.u.)

\begin{tabular}{llllll}
\hline & $\begin{array}{l}\text { Simple } \\
\text { Tariff }\end{array}$ & $\begin{array}{l}\text { Bi-hourly tariff } \\
\text { (day cycle) }\end{array}$ & $\begin{array}{l}\text { Bi-Hourly tariff } \\
\text { (week cycle) }\end{array}$ & $\begin{array}{l}\text { Tri-Hourly tariff } \\
\text { (day cycle) }\end{array}$ & $\begin{array}{l}\text { Tri-Hourly tariff } \\
\text { (week cycle) }\end{array}$ \\
\hline Library PV & $27,994.55$ & $27,711.77$ & $26,684.38$ & $28,276.23$ & $27,053.75$ \\
\% of savings & 13.86 & 16.40 & 15.50 & 16.86 & 15.75 \\
Municipal Market PV & 5235.50 & 4301.23 & 4506.00 & 4461.75 & 4397.37 \\
\% of savings & 46.24 & 55.83 & 52.06 & 56.24 & 53.49 \\
Culture House PV & $33,839.73$ & $30,125.91$ & $30,193.33$ & $31,076.41$ & $29,066.71$ \\
\% of savings & 22.73 & 28.57 & 26.31 & 28.39 & 27.79 \\
Total cost & $67,069.78$ & $62,138.91$ & $61,383.72$ & $63,814.39$ & $60,517.82$ \\
\% of savings & 22.04 & 26.95 & 25.10 & 27.15 & 26.03 \\
\hline
\end{tabular}

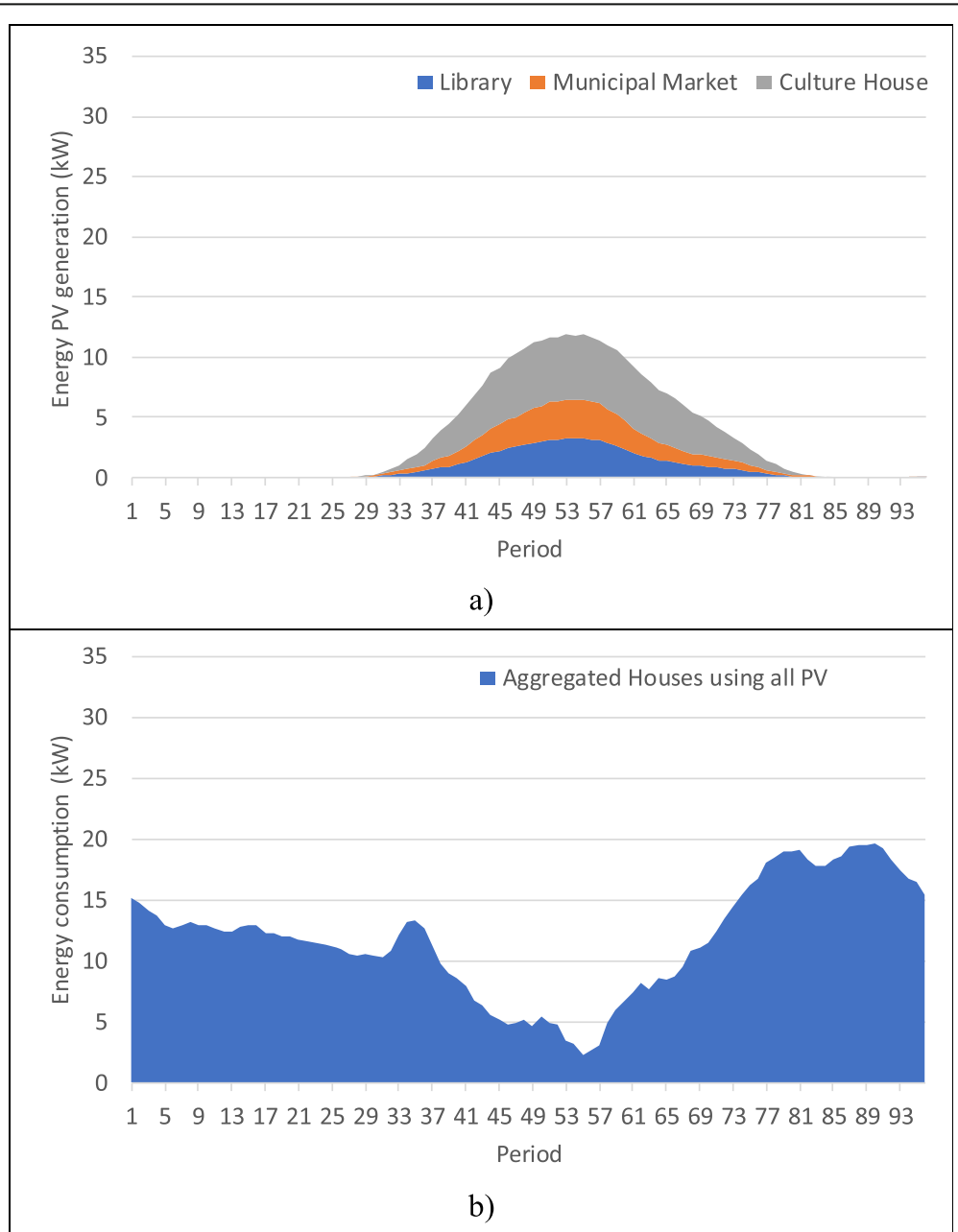

Fig. 4 Average PV generation and the impact in using that generation for the residential houses of the community. a Aggregated PV generation. b Aggregated consumption of the residential houses after using the available PV generation 
Table 3 Electricity Annual cost and percentage of savings for residential houses with distinct types of tariffs if PV generation is used (m.u.)

\begin{tabular}{llllll}
\hline & $\begin{array}{l}\text { Simple } \\
\text { Tariff }\end{array}$ & $\begin{array}{l}\text { Bi-hourly tariff } \\
\text { (day cycle) }\end{array}$ & $\begin{array}{l}\text { Bi-Hourly tariff } \\
\text { (week cycle) }\end{array}$ & $\begin{array}{l}\text { Tri-Hourly tariff } \\
\text { (day cycle) }\end{array}$ & $\begin{array}{l}\text { Tri-Hourly tariff } \\
\text { (week cycle) }\end{array}$ \\
\hline Agg. houses using PV & $66,777.64$ & $62,288.26$ & $61,890.84$ & $65,121.38$ & $60,193.21$ \\
\% of savings & 22.12 & 26.90 & 24.95 & 26.76 & 26.14 \\
\hline
\end{tabular}

generation impact. We can provide a general analysis between the baseline (i.e., consumption patterns and annual cost with no PV generation) and the use of installed PV capacity. Figure 5 shows the annual distribution of power generation and consumption. Figure 5a shows that the culture house provides more than 50\% of PV generation. Another interesting point can be observed in Fig. 5b, where it is showed that public buildings present a consumption of $58 \%$ of the energy community, surpassing the overall consumption of all residential houses.

Table 4 concludes the analysis, reporting the total annual costs using the different available tariffs and the total savings obtained by using the PV generation in the community. As can be seen, around 9-11\% of total savings can be obtained using PV generation for this case study. Depending on the selection of different tariff, the total costs sharing the PV generation oscillates between approximately 175,000 m.u. and 185,000 m.u.

\section{Conclusions}

In this paper, a KPIs analysis methodology was presented to evaluate the impact of energy management solutions. A methodology based on the use of KPI was proposed as a framework to determine a baseline and assess the impact of different KPIs. The methodology allows the use of KPIs to abstract the information obtained by power measurements into quantifiable data of natural interpretation. With the implemented case study, it was possible to see how the KPI of the energy bill (and savings) changes in 1 year by installing PV generation in public buildings and ideally changing the energy

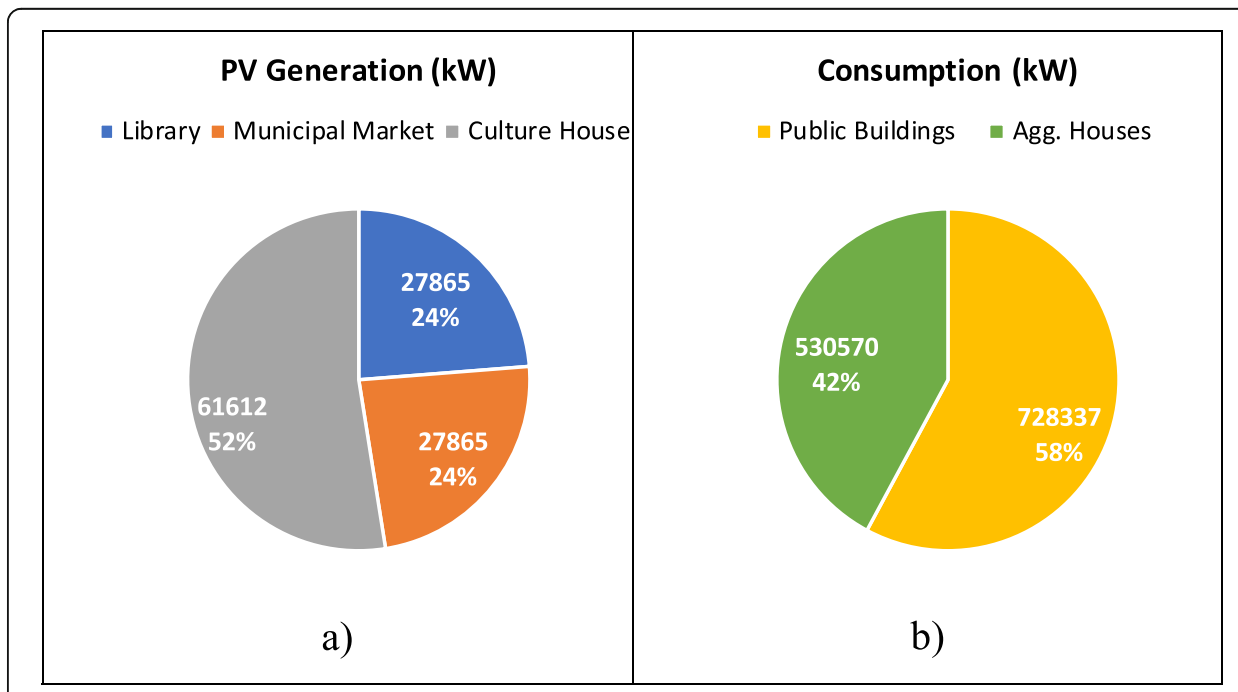

Fig. 5 Total distribution of PV generation and consumption. a Annual PV generation in kW and percentage distribution. b Annual power consumption of aggregated houses and aggregated public buildings 
Table 4 Savings to share in the community

\begin{tabular}{llllll}
\hline & Simple & $\begin{array}{l}\text { Bi-hourly } \\
\text { (day cycle) }\end{array}$ & $\begin{array}{l}\text { Bi-Hourly } \\
\text { (week cycle) }\end{array}$ & $\begin{array}{l}\text { Tri-Hourly } \\
\text { (day cycle) }\end{array}$ & $\begin{array}{l}\text { Tri-Hourly } \\
\text { (week cycle) }\end{array}$ \\
\hline Total cost without PV & $203,439.48$ & $203,794.30$ & $196,136.47$ & $210,979.90$ & $195,655.48$ \\
PV generation & $18,962.55$ & $22,923.41$ & $20,570.21$ & $23,787.95$ & $21,301.47$ \\
Total cost after PV & $184,476.93$ & $180,870.89$ & $175,566.25$ & $187,191.96$ & $174,354.01$ \\
\% savings & 9.32 & 11.25 & 10.49 & 11.27 & 10.89 \\
\hline
\end{tabular}

tariff to better match the one that better fits the PV generation profile. Using the proposed simple methodology, evaluating the energy bill KPI with different tariffs and PV generation along time was possible, while in the previous studies, the results are compared only at the end of the day or at the end of the month or year. Results showed that savings up to $11.27 \%$ can be achieved by using and sharing the PV generation. Moreover, the application of different tariffs over the same consumption patterns can be reflected in annual costs and savings. The application presented in the case study is a rather simple one for illustration purposes. In fact, the developed methodology can be applied in different contexts to evaluate the value of different energy management projects, driven to an online KPI evaluation tool that dynamically provides information to the users about the impact of energy management measures impact.

Acknowledgements

Not applicable.

Authors' contributions

FL researched on the existing KPIs. PF and ZV developed the methodology. ZV raised and developed the overall idea of the work. MK calculated the KPIs in the case study. All authors read and approved the final manuscript.

Funding

This work has received funding from FEDER Funds through COMPETE program and from National Funds through (FCT) under the projects UIDB/00760/2020, COLORS (PTDC/EEIEEE/28967/2017), and CEECIND/02887/2017.

Availability of data and materials

Please contact author for data requests.

\section{Declarations}

Ethics approval and consent to participate

Not applicable.

\section{Consent for publication}

Not applicable.

Competing interests

The authors declare that they have no competing interests.

\section{Author details}

${ }^{1}$ GECAD-Research Group on Intelligent Engineering and Computing for Advanced Innovation and Development, Porto, Portugal. ${ }^{2}$ Polytechnic of Porto, Porto, Portugal.

Received: 30 November 2020 Accepted: 18 March 2021

Published online: 12 April 2021

References

Abrishambaf O, Gomes L, Faria P, Vale Z (2015) Simulation and control of consumption and generation of hardware resources in microgrid real-time digital simulator. In: 2015 IEEE PES innovative smart grid technologies Latin America (ISGT LATAM), pp 799-804

Al Dakheel, Del Pero, Aste N, Leonforte F (2020) Smart buildings features and key performance indicators: a review. Sustain Cities Soc 61:102328. https://doi.org/10.1016/j.scs.2020.102328

Atwa YM, El-Saadany EF (2009) Reliability evaluation for distribution system with renewable distributed generation during islanded mode of operation. IEEE Trans Power Syst 24(2):572-581. https://doi.org/10.1109/TPWRS.2009.2016458 
Cabeza LF, Galindo E, Prieto C, Barreneche C, Inés Fernández A (2015) Key performance indicators in thermal energy storage: survey and assessment. Renew Energy 83:820-827. https://doi.org/10.1016/j.renene.2015.05.019

Del Pero, Aste N, Paksoy H, Haghighat F, Grillo S, Leonforte F (2018) Energy storage key performance indicators for building application. Sustain Cities Soc 40:54-65. https://doi.org/10.1016/j.scs.2018.01.052

ERSE, (n.d.) "Regulamento de Tarifário - Setor Elétrico", [In Portuguese], available online: https://www.erse.pt/media/ bhnpuida/articulado-rt-se_consolidado.pdf. Accessed Dec 2019.

Fan Z, Kulkarni P, Gormus S, Efthymiou C, Kalogridis G, Sooriyabandara M, Zhu Z, Lambotharan S, Chin WH (2013) Smart grid communications: overview of research challenges, solutions, and standardization activities. IEEE Commun Surv Tutorials 15(1):21-38. https://doi.org/10.1109/SURV.2011.122211.00021

Faria P, Barreto R, Vale Z (2019) Demand response in energy communities considering the share of photovoltaic generation from public buildings. International conference on smart energy systems and technologies (SEST), Porto, pp 1-6. https:// doi.org/10.1109/SEST.2019.8849082

Giordano V, Vitiello S, Vasiljevska J (2014) Definition of an assessment framework for projects of common interest in the field of smart grids. JRC Sci Policy Rep. https://doi.org/10.2790/83888

González-Gil A, Palacin R, Batty P (2015) Optimal energy management of urban rail systems: key performance indicators. Energy Convers Manag 90:282-291. https://doi.org/10.1016/j.enconman.2014.11.035

Grigsby LL (2012) Power systems, 3rd edn

Hanak DP, Kolios AJ, Biliyok C, Manovic V (2015) Probabilistic performance assessment of a coal-fired power plant. Appl Energy 139:350-364. https://doi.org/10.1016/j.apenergy.2014.10.079

Honarmand N (2015) Key performance indicators modeling for optimized microgrid configuration

Hossam NH, Gabbar A, Xiaoli M, Abdelsalam A (2014) Key performance indicator modeling for micro grid design and operation evaluation. Int J Distrib Energy Resour Smart Grids 10(4):219-242

IPMVP (2001) International performance measurement and verification protocol: concepts and options for determining energy and water savings, volume I

Kourkoumpas D-S, Benekos G, Nikolopoulos N, Karellas S, Grammelis P, Kakaras E (2018) A review of key environmental and energy performance indicators for the case of renewable energy systems when integrated with storage solutions. Appl Energy 231:380-398. https://doi.org/10.1016/j.apenergy.2018.09.043

Lezama F, Palominos J, Rodriguez-Gonzalez AY, et al (2019) Agent-based microgrid scheduling: an ICT perspective. Mob Networks Appl 24:1682-1698. https://doi.org/10.1007/s11036-017-0894-x

Li W, Logenthiran T, Woo WL (2015) Intelligent multi-agent system for smart home energy management. In: Smart grid Technol. - Asia (ISGT ASIA). IEEE Innov, pp 1-6

Li Y, O'Donnell J, García-Castro R, Vega-Sánchez S (2017) Identifying stakeholders and key performance indicators for district and building energy performance analysis. Energy Build 155:1-15. https://doi.org/10.1016/j.enbuild.2017.09.003

May G, Barletta I, Stahl B, Taisch M (2015) Energy management in production: a novel method to develop key performance indicators for improving energy efficiency. Appl Energy 149:46-61. https://doi.org/10.1016/j.apenergy.2015.03.065

Moretti M, Djomo SN, Azadi H, May K, de Vos K, van Passel S, Witters N (2017) A systematic review of environmental and economic impacts of smart grids. Renew Sust Energ Rev 68:888-898. https://doi.org/10.1016/j.rser.2016.03.039

Nistor M, Antunes C (2016) Integrated Management of Energy Resources in residential buildings - a Markovian approach. IEEE Transact Smart Grid PP(99):1

Peng Z, Suryanarayanan S, Simoes MG (2013) An energy management system for building structures using a multi-agent decision-making control methodology. Ind Appl IEEE Trans 49(1):322-330

Personal E, Guerrero Jl, Garcia A, Peña M, Leon C (2014) Key performance indicators: a useful tool to assess smart grid goals. Energy 76:976-988. https://doi.org/10.1016/j.energy.2014.09.015

Pramangioulis D, Atsonios K, Nikolopoulos N, Rakopoulos D, Grammelis P, Kakaras E (2019) A methodology for determination and definition of key performance indicators for smart grids development in island energy systems. Energies 12(2):242. https://doi.org/10.3390/en12020242

Soares JAP (2017) Energy resource management in smart grids with intensive use of electric vehicles: heuristic and deterministic approaches

Van Gorp (2005) Using key performance indicators to manage energy costs. Strateg Plan Energy Environ 25(2):9-25. https:// doi.org/10.1080/10485230509509683

Yang H, Wei Z, Chengzhi L (2009) Optimal design and techno-economic analysis of a hybrid solar-wind power generation system. Appl Energy 86(2):163-169. https://doi.org/10.1016/j.apenergy.2008.03.008

\section{Publisher's Note}

Springer Nature remains neutral with regard to jurisdictional claims in published maps and institutional affiliations. 